\title{
A method for deriving normal ranges from laboratory specimens applied to uric acid in males
}

\author{
M. G. COOK, M. J. LEVELL, AND R. B. PAYNE
}

From the Department of Chemical Pathology, School of Medicine, Leeds

SYNOPSIS Plasma specimens from male blood donors gave a normal range for uric acid concentration of 3.3 to $7.5 \mathrm{mg} / 100 \mathrm{ml}$. Specimens from male outpatients, which were received in the laboratory for the analysis of urea but not of uric acid and proved to have normal concentrations of urea, showed a positively skewed distribution of values for uric acid from which a 'normal range' of 3.2 to $7.6 \mathrm{mg} / 100 \mathrm{ml}$ was derived. We suggest that specimens from outpatients selected in the way described here may prove to be a convenient source of data for determining the normal range of other substances.

Ideally, normal ranges for measurements which are of specific diagnostic value should be determined on patients with some of the signs or symptoms of the disease being considered but who do not in fact have that disease because they form the 'control' group from which a patient with the disease must be distinguished (Payne and Levell, 1968). This ideal is difficult to achieve. Normal ranges for a number of different determinations have been derived from the bulk of laboratory results on unselected patients by extracting an appropriate distribution by mathematical methods (Pryce, 1960; Hoffmann, 1963; Amador and Hsi, 1969). However, before such methods are applied to a determination it is essential to examine the assumption which is implicit in them that only a minority of the values are of diagnostic significance.

An examination of laboratory data for uric acid showed, as it had for sodium (Payne and Levell, 1968), that the results of analyses which had been requested on patients included too many abnormal values for a useful range to be derived from them mathematically. However, again as with sodium, a useful range for uric acid could be derived from the values obtained by the prospective analysis of specimens received in the Received for publication 30 April 1970. laboratory from outpatients on whom the specific investigation had not been requested.

\section{Analytical Methods}

Plasma uric acid was measured by the AutoAnalyzer sodium tungstate method (Technicon Methodology N-13a) modified by substituting sodium carbonate for sodium cyanide (Caraway, 1955). Uric acid concentrations were recorded to the nearest $0.1 \mathrm{mg} / 100 \mathrm{ml}$. The monthly coefficients of variation of analyses of pooled frozen sera for uric acid at values near $6.0 \mathrm{mg} / 100 \mathrm{ml}$ had a mean of $3.6 \%$ with a range from 2.5 to $5.0 \%$, and single standard quality control charts and the analysis of control sera showed that there was no significant change in the accuracy of the method over the period of the study.

\section{Selection of Specimens}

Plasma specimens with urea concentrations less than $43 \mathrm{mg} / 100 \mathrm{ml}$ were used in order to exclude renal failure which is known to raise uric acid concentrations. No measures were taken to 
exclude specimens from patients with other conditions known to alter the plasma uric acid concentration because we wished to use only information available on the request form. Four groups of specimens from men were studied.

1 A series of 109 specimens from blood donors taken during a single session.

2 A series of 87 consecutive specimens received in the laboratory for the analysis of uric acid.

3 A series of 101 specimens from inpatients received for the analysis of sodium, potassium, chloride, $\mathrm{CO}_{2}$-combining power, or urea. (These five analyses are done simultaneously on a single specimen in this laboratory.)

4 A series of 96 specimens from outpatients received for electrolyte or urea analysis.

\section{Results}

When the results of group 1, the blood donors, were plotted on arithmetic and log probability paper it was clear that they approximated more closely to a Gaussian distribution than to a logarithmic distribution. The calculated $95 \%$ normal range (mean $\pm 2 s$ ) was $3 \cdot 34-7 \cdot 46$ $\mathrm{mg} / 100 \mathrm{ml}$ (Table I).

The group 2 results gave a wide flat distribution, ranging from 2.8 to $13.8 \mathrm{mg} / 100 \mathrm{ml}$, with no clear bulk population of values.

The group 3 results showed a bulk population with a tail of higher values. However, when the values below the mode were plotted on arithmetic probability paper they did not give a straight line but showed an excess of low values as well.

The group 4 results also showed a bulk population of values but, in contrast to the inpatient data, the values below the mode gave a reasonably straight line on arithmetic probability paper. Moreover, the line was close to that which fitted the blood donor data. The distribution derived from this group using the convention of Pryce (1960) gave a range which was close to the blood donor range (Table I). We preferred Pryce's convention for the analysis of our prospective outpatient data to the simple alternative methods (eg, Hoffmann, 1963) because it is completely objective and does not involve drawing the best line through a series of points.

There was no significant correlation of the plasma uric acid concentration in any of the groups studied either with age or with urea concentration, the urea values all lying in the? range $14-42 \mathrm{mg} / 100 \mathrm{ml}$.

\section{Discussion}

A variety of mathematical procedures have been proposed for deriving normal ranges from $\vec{\omega}$ laboratory results. These have been criticallys reviewed by Amador and Hsi (1969). Sucho methods are useful only when the proportion of diagnostically abnormal values is known to beio very low, as for example with the excretion of 4-hydroxy-3-methoxymandelic acid (HMMA) in urine (Payne and Levell, unpublished data), ? but are inapplicable when the clinicians are highly selective in their requests and the proportion ofo abnormal values is likely to be high.

We suggest a way around this difficulty which? combines methods of selecting specimens appli- $\frac{\Phi}{\mathrm{D}}$ cable within the laboratory with mathematical techniques. Specimens are taken only from out-patients so as to exclude most serious illnesses. and outpatient specimens on which the particularo analysis has been requested are rejected to excludes those from many patients in whom there is a clinical suspicion of diseases known to affect theo value. In the present investigation it was also possible specifically to exclude specimens from $\overrightarrow{\overrightarrow{0}}$ patients with renal failure by measuring the ${ }^{3}$ plasma urea. The remaining outpatient specimens are analysed prospectively and the resulting. distribution of values is mathematically curtailed using the convention of Pryce (1960) if there is $a$ strong presumption that the remaining abnormaB values are all either high or low. Using this method we derived from outpatient specimens a normaB range for plasma uric acid in males of 3.20 too $7.60 \mathrm{mg} / 100 \mathrm{ml}$, only a little wider than the blood donor normal range of 3.34 to $7.46 \mathrm{mg} / 100 \mathrm{mro}$ (Table I).

O'Halloran, Studley-Ruxton, and Wellby (1970) have recently published a comparison of

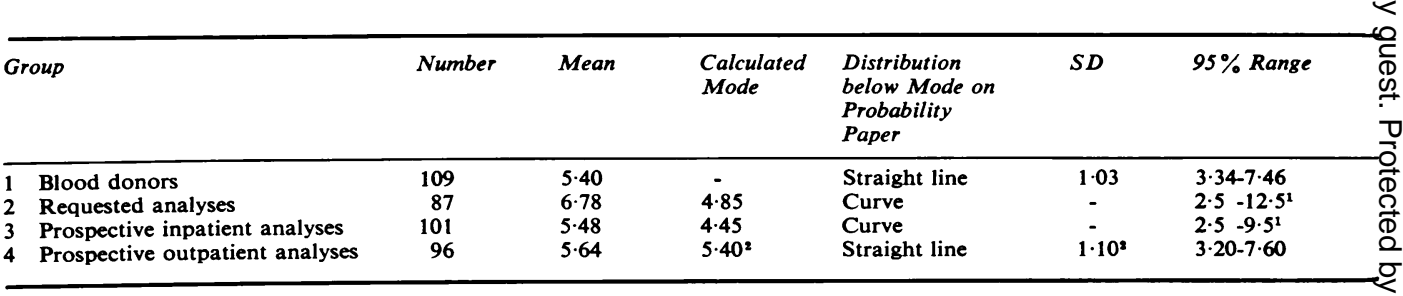

Table I Plasma uric acid concentration in males $(\mathrm{mg} / 100 \mathrm{ml})$ 
the distribution of the results of 12 laboratory tests on specimens from inpatients, outpatients, and healthy persons. They used a 12-channel sequential multiple analyser (Technicon hospital model SMA-12) so it is probable that the majority of the tests performed, particularly on outpatients, would not ordinarily have been requested by the clinician, but may be regarded as prospective analyses. For many tests the normal ranges derived from the analyses on outpatient specimens were very close to the ranges derived from analyses on specimens from laboratory and clinical staff. These findings lead us to believe that our method of selecting outpatient specimens on which the analysis has not been requested-a method applicable to all ages and to urine as well as blood-will prove to be a useful alternative to blood donor specimens as a source of normal ranges.
The analyses were carried out in the Department' $\overline{\bar{S}}$ Clinical Biochemistry Laboratory. We thank Mis Eileen Davy and Miss Pauline Jolliff who bore the brunt of the extra analytical work.

Requests for reprints should be addressed to R.B.P.

References

Amador. E., and Hsi, B. P. (1969). Indirect methods for estimatin the normal range. Amer. J. clin. Path., 52, 538-546.

Caraway, W. T. (1955). Determination of uric acid in serum by छొ carbonate method. Amer. J. clin. Path., 25, 840-845.

Hoffmann, R. G. (1963). Statistics in the practice of medicine $\overrightarrow{0}$ $J$. Amer. med. Ass., 185, 864-873.

O'Halloran, M. W., Studley-Ruxton, J., and Wellby, M. L. (1970) A comparison of conventionally derived normal ranges with those obtained from patients' results. Clin. chim. Acta 27, 35-46.

Payne, R. B., and Levell, M. J. (1968). Redefinition of the normat range for serum sodium. Clin. Chem., 14, 172-178.

Pryce, J. D. (1960). Level of haemoglobin in whole blood and redo blood-cells, and proposed convention for defining nor: mality. Lancet, 2, 333-336. 